\title{
Comunicação/Communication
}

\section{Destruição de ovos de Toxocara canis pelo fungo nematófago Pochonia chlamydosporia}

\author{
Destruction of Toxocara canis eggs by the nematophagous fungus Pochonia chlamydosporia
}

\section{Luiza NemeFrassy ${ }^{1}$, Fabio Ribeiro Braga ${ }^{1}$, André Ricardo eSilva ${ }^{1}$, Jackson Victor de Araújo ${ }^{1}$,Sebastião Rodrigo Ferreira ${ }^{1}$ e Leandro Grassi de Freitas ${ }^{2}$}

\section{RESUMO}

Introdução: Toxocara canis é um ascarídeo parasita do intestino delgado de cães, causador da larva migrans visceral em seres humanos. Métodos: Com o objetivo de demonstrar a eficácia do fungo Pochonia chlamydosporia sobre ovos de Toxocara canis em condições laboratoriais, foi montado ensaio experimental em placas de Petri com ágar-água $2 \%$. Resultados: Houve atividade ovicida de $43,8 \%(p<0,01)$ do grupo tratado em relação ao grupo controle durante os intervalos estudados. Conclusões: Os resultados demonstrados no presente trabalho sugerem a empregabilidade de Pochonia chlamydosporia como uma alternativa de controle biológico dos ovos embrionados de Toxocara canis.

Palavras-chaves: Fungos nematófagos. Pochonia chlamydosporia. Toxocara canis.

\begin{abstract}
Introduction: Toxocara canis is an ascarid parasite of the small intestine of dogs that causes visceral larva migrans in humans. Methods: With the aim of demonstrating the effectiveness of the fungus Pochonia chlamydosporia on Toxocara canis eggs under laboratory conditions, a trial was set up in Petri dishes with $2 \%$ agar-water. Results: There was ovicidal activity of $43.8 \%$ (p $<0.01$ ) in the treated group in relation to the control group over the periods studied. Conclusions: The results from the present study suggest that Pochonia chlamydosporia can potentially be used as an alternative biological control for embryonated Toxocara canis eggs.
\end{abstract}

Key-words: Nematophagous fungi. Pochonia chlamydosporia. Toxocara canis.

Os estudos sobre parasitismo em animais de estimação vêm despertando crescente interesse, frente à associação restrita e íntima entre o homem e os animais e sua consequência em saúde pública ${ }^{1}$. Toxocara canis é um ascarídeo parasita do intestino delgado de cães, com distribuição cosmopolita ${ }^{2,3}$.

Os casos de larva migrans visceral são ocasionados frequentemente por Toxocara canis e Toxocara cati, por ingestão de ovos embrionados do ambiente. Seres humanos e outros mamíferos quando infectados

1. Departamento de Veterinária, Universidade Federal de Viçosa, Viçosa, MG. 2. Departamento de Fitopatologia, Universidade Federal de Viçosa, Viçosa, MG.

Endereço para correspondência: Dr. Fabio R. Braga. Dept ${ }^{\circ}$ de Veterinária/ UFV. Av. Ph Rolfes s/n, 36570-000 Viçosa, MG.

Tel: 5531 3899-1458.

e-mail: fabioribeirobraga@hotmail.com

Recebido para publicação em 06/07/2009

Aceito em 10/12/2009 por larvas de Toxocara canis comportam-se como hospedeiros paratênicos, não permitindo o desenvolvimento completo do helminto. As larvas, contudo, podem sobreviver por longos períodos no organismo humano, realizando migrações por órgãos e tecidos, onde permanecem encistadas e viáveis, podendo determinar manifestações clínicas diversas, que caracterizam a síndrome de larva migrans visceral, a larva migrans ocular e a toxocaríase oculta ${ }^{4}$.

Segundo Barriga ${ }^{2}$, algumas medidas de prevenção da toxocaríase seriam o controle da população canina, a educação do público sobre o potencial zoonótico desse nematóide e a limitação do acesso de animais a áreas de lazer. Por outro lado, a alta resistência dos ovos no ambiente e a dificuldade de desinfecção justificam a necessidade da implementação de medidas alternativas que ajudem na descontaminação do solo, sendo essa a principal fonte de contaminação ${ }^{3,5}$. Entre elas, está o uso de fungos nematófagos que estão presentes no meio ambiente e, cuja ação está concentrada no ambiente fecal e direcionada ao combate dos ovos e das larvas de vida livre dos geohelmintos. Dentre esses fungos, destaca-se a espécie e Pochonia chlamydosporia considerada ovicida, pois causa a destruição de ovos por conseqüência do seu parasitismo ${ }^{6}$.

$\mathrm{O}$ presente estudo objetivou avaliar in vitro a ação ovicida do fungo nematófago Pochonia chlamydosporia (isolados VC1, VC4, VC5, VC12 e VC31) sobre ovos de Toxocara canis em diferentes intervalos de tempo.

Cinco isolados do fungo nematófago Pochonia chlamydosporia (VC1, VC4, VC5, VC12 e VC31) foram mantidos em tubos de ensaio contendo o meio de cultura Corn-Meal-Agar $2 \%$, no escuro a $4^{\circ} \mathrm{C}$ por 10 dias. Dois isolados (VC1 e VC4) são provenientes do Laboratório de Parasitologia da Universidade Federal de Viçosa (UFV), MG, Brasil. E os outros três (VC5, VC12 e VC31) são provenientes do laboratório de Controle Biológico de Nematóides do Departamento de Fitopatologia da UFV.

Ovos de Toxocara canis foram obtidos pela dissecção do útero de fêmeas adultas provenientes de um cão jovem parasitado, morto por causas naturais. A identificação dos exemplares adultos e dos ovos foi realizada com base nas descrições de Urquhart e cols ${ }^{3}$. Os ovos foram lavados 10x em água destilada por centrifugação a 1.000rpm por 5 minutos cada vez, desprezando o sobrenadante ao fim de cada centrifugação. Posteriormente, os ovos foram incubados a $25^{\circ} \mathrm{C}$ por 14 dias em solução contendo $0,05 \%$ de formalina, $0,005 \%$ de sulfato de estreptomicina e $0,01 \%$ de cloranfenicol. Após este período, foi repetido o processo de lavagem em água destilada como descrito acima.

Ovos de Toxocara canis foram analisados morfologicamente quanto a sua integridade por meio de microscopia óptica, objetiva de $10 \mathrm{x}$, seguindo os critérios descritos por Araújo cols ${ }^{7}$. A seguir, 
foram vertidos sobre a superfície das placas de Petri de $9 \mathrm{~cm}$ de diâmetro contendo apenas o meio ágar-água $2 \%$ com isolados fúngicos crescidos por 10 dias e sem fungo como controle, em temperatura de $26^{\circ} \mathrm{C}$, sendo feitas 10 repetições para cada grupo. Nos tratamentos, cada placa de Petri continha mil ovos de Toxocara canis com apenas um dos isolados fúngicos emil ovos sem fungo (controle). Nos intervalos de cinco, 10 e 15 dias, cerca de cem ovos foram retirados de cada placa contendo o isolado fúngico e do controle (sem fungo) segundo a técnica descrita por Araújo e cols ${ }^{2}$ e, colocados em lâminas de vidro com uma gota de azul de Amam 1\%.Após isso, os ovos foram avaliados por meio de microscopia de luz em objetiva de 40x de acordo com os parâmetros estabelecidos por Lysek cols ${ }^{8}$ : tipo 1, efeito fisiológico, bioquímico sem prejuízo morfológico à casca do ovo, onde hifas são observadas aderidas à casca; tipo 2, efeito lítico com alteração morfológica da casca e embrião do ovo, sem penetração de hifas através da casca; e tipo 3 efeito lítico com alteração morfológica do embrião e da casca, além de penetração de hifas e colonização interna do ovo.

Os dados do ensaio experimental ao longo dos intervalos foram submetidos ao teste não paramétrico de Friedman com $1 \%$ de probabilidade?.

Os resultados percentuais para os efeitos tipo 1, 2 e 3 aos cinco, 10 e 15 dias para os grupos tratados com os fungos Pochonia chlamydosporia (isolados VC4, VC31, VC1, VC12 e VC5 e para o grupo controle estão representados na Tabela 1. Durante o presente trabalho, o fungo Pochonia chlamydosporia (VC4, VC31, VC1, VC12 e VC5) demonstrou resultados percentuais de ação ovicida (efeito do tipo 3) sobre os ovos de Toxocara canis em todos os intervalos estudados. No grupo controle, não foi constatado a presença de fungos. No grupo tratado com o fungo Pochonia chlamydosporia (VC4, VC31, VC1, VC12 e VC5) foi observada a presença de ovos rompidos a partir do $15^{\circ}$ dia de interação (Figura 1).

O solo de praças e parques públicos constitui via de transmissão para zoonoses parasitárias ${ }^{10}$. Segundo Bourdeau ${ }^{11}$, ovos de Toxocara canis, devido à consistência de sua cutícula externa, permanecem viáveis por longo período no ambiente, expondo a população humana ao risco de infecção desenvolvimento da doença. No presente trabalho, demonstrou-se que a ação de Pochonia chlamydosporia se manteve durante o experimento, sendo responsável pela destruição dos ovos ao final de 15 dias de interação. Esta informação é importante, pois segundo Overgaauw ${ }^{12}$ e Urquhart e cols ${ }^{3}$ os ovos de Toxocara canis atingiriam seu desenvolvimento em torno de 2 a 6 semanas tornando-se infectantes.
TABELA 1 - Percentual de atividade ovicida e desvios padrão do fungo nematófago Pochonia chlamydosporia (VC4, VC31, VC1, VC12 e VC5), e o grupo controle sem fungos, sobre ovos de Toxocara canis nos intervalos de 5,10 e 15 dias.

\begin{tabular}{|c|c|c|c|}
\hline \multirow[b]{2}{*}{ Isolados } & \multicolumn{3}{|c|}{5 dias de interação } \\
\hline & efeito do tipo $1^{*}$ & efeito do tipo $2^{* *}$ & efeito do tipo $3^{* * *}$ \\
\hline VC4 & $47,7^{\mathrm{A}} \pm 17,01$ & $26,9^{A} \pm 7,99$ & $25,4^{\mathrm{A}} \pm 10,95$ \\
\hline VC31 & $54^{A} \pm 14,62$ & $24,8^{\mathrm{A}} \pm 11,66$ & $21,2^{\mathrm{A}} \pm 7,74$ \\
\hline VC1 & $32,5^{A} \pm 32,55$ & $29,6^{\mathrm{A}} \pm 14,73$ & $39,9^{\mathrm{A}} \pm 35,13$ \\
\hline VC12 & $39,9^{A} \pm 11,67$ & $42^{\mathrm{A}} \pm 9,74$ & $18,1^{A} \pm 16,23$ \\
\hline VC5 & $63,7^{\mathrm{A}} \pm 8,21$ & $24,8^{A} \pm 9,23$ & $10,5^{\mathrm{A}} \pm 7,87$ \\
\hline \multirow[t]{2}{*}{ Controle } & $0^{\mathrm{B}} \pm 0$ & $0^{\mathrm{B}} \pm 0$ & $0^{\mathrm{B}} \pm 0$ \\
\hline & \multicolumn{3}{|c|}{10 dias de interação } \\
\hline Isolados & efeito do tipo $1^{*}$ & efeito do tipo $2^{* *}$ & efeito do tipo $3^{* * *}$ \\
\hline VC4 & $4,2^{A} \pm 4,39$ & $19,1^{\mathrm{A}} \pm 11,40$ & $76^{\mathrm{A}} \pm 11,90$ \\
\hline VC31 & $2^{A} \pm 2,58$ & $55^{\mathrm{B}} \pm 12,37$ & $43^{\mathrm{B}} \pm 11,98$ \\
\hline VC1 & $8,2^{A} \pm 12,09$ & $40,7^{\mathrm{A}, \mathrm{B}} \pm 19,87$ & $53,6^{\mathrm{B}} \pm 21,45$ \\
\hline $\mathrm{VC} 12$ & $2^{A} \pm 2,94$ & $43,2^{\mathrm{B}} \pm 19,35$ & $54^{\mathrm{A}, \mathrm{B}} \pm 19,42$ \\
\hline VC5 & $0,1^{A} \pm 0,31$ & $46,8^{\mathrm{B}} \pm 30,35$ & $59^{\mathrm{A}, \mathrm{B}} \pm 25,24$ \\
\hline \multirow[t]{2}{*}{ Controle } & $0^{\mathrm{B}} \pm 0$ & $0^{\mathrm{C}} \pm 0$ & $0^{\mathrm{C}} \pm 0$ \\
\hline & \multicolumn{3}{|c|}{15 dias de interação } \\
\hline Isolados & efeito do tipo $1^{*}$ & efeito do tipo $2^{* *}$ & efeito do tipo $3^{* * *}$ \\
\hline $\mathrm{VC} 4$ & $15^{A} \pm 13,00$ & $34,8^{A} \pm 9,65$ & $47,3^{A} \pm 12,73$ \\
\hline VC31 & $0,6^{A} \pm 1,34$ & $46,9^{A} \pm 12,74$ & $52,5^{\mathrm{A}} \pm 13,13$ \\
\hline VC1 & $24^{\mathrm{A}, \mathrm{B}} \pm 15,43$ & $32,{ }^{\mathrm{A}, \mathrm{B}} \pm 9,86$ & $43,1^{A} \pm 8,64$ \\
\hline $\mathrm{VC} 12$ & $26,1^{\mathrm{A}, \mathrm{B}} \pm 19,82$ & $35^{A} \pm 9,85$ & $39^{A} \pm 12,09$ \\
\hline VC5 & $34^{\mathrm{B}} \pm 15,13$ & $29,1^{\mathrm{A}, \mathrm{B}} \pm 9,48$ & $36,9^{\mathrm{A}, \mathrm{B}} \pm 11,70$ \\
\hline Controle & $0^{\mathrm{C}} \pm 0$ & $0^{\mathrm{C}} \pm 0$ & $0^{\mathrm{C}} \pm 0$ \\
\hline
\end{tabular}

Percentuais seguidos da mesma letra maiúscula não diferem $(\mathrm{P}>0,01)$ - Teste de Friedman.

*Efeito do tipo 1, efeito fisiológico, bioquímico sem prejuízo morfológico à casca do ovo, onde hifas são observadas aderidas à casca; ${ }^{* *}$ Efeito do tipo 2, efeito lítico com alteração morfológica da casca e embrião do ovo, sem penetração de hifas através da casca; ${ }^{* * *}$ Efeito do tipo 3, efeito lítico com alteração morfológica do embrião e da casca, além de penetração de hifas e colonização interna do ovo.

Braga e cols ${ }^{6}$ demonstraram a eficácia dos isolados VC1 e VC4 do fungo Pochonia chlamydosporia sobre ovos de Ascaris lumbricoides, nematóide gastrintestinal comum de seres humanos, nos intervalos de sete, 10 e 14 dias, e ao final do experimento estes autores registraram percentuais para a ação ovicida acima de $26 \%$ para ambos os isolados testados. Contudo, quando esse efeito é comparado com os resultados

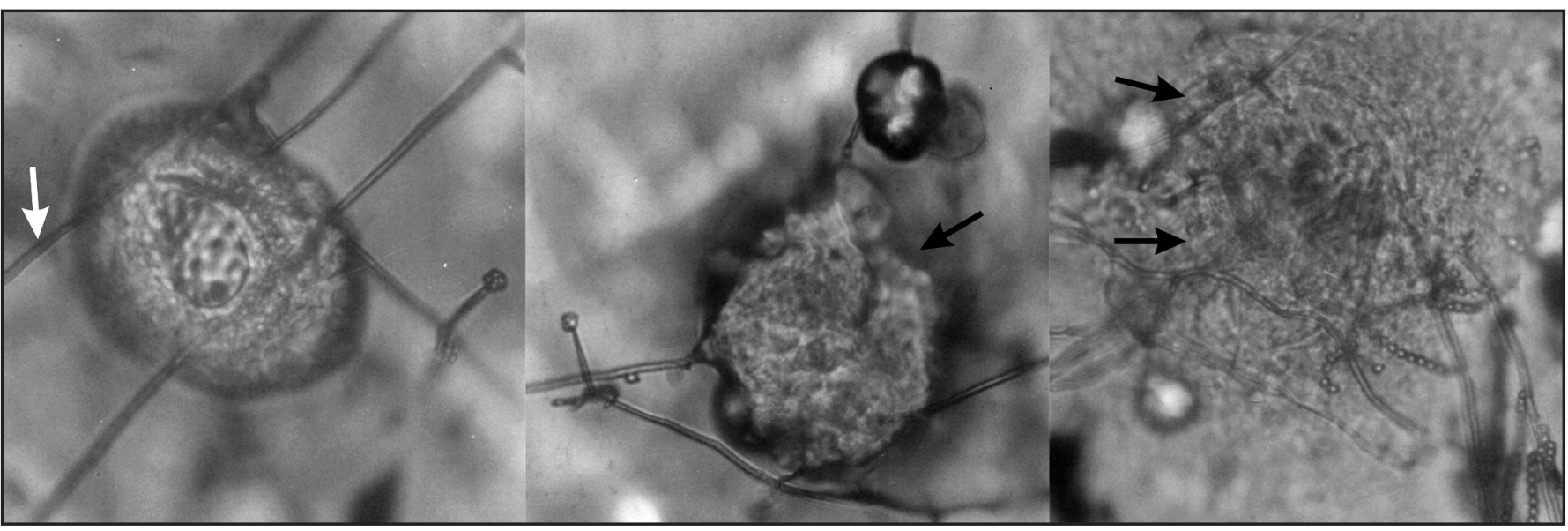

FIGURA 1- Hifas do fungo Pochonia chlamydosporia (seta branca) e ovo destruído de Toxocara canis (seta preta) aos 15 dias de interação. Microscopia óptica com aumento de 40x. 
do presente trabalho ao final de 15 dias, nota-se que sua ação foi maior sobre ovos de Toxocara canis cujos valores percentuais correspondem a $43,1 \%$ e $47,3 \%$ para VC1 e VC4, respectivamente.

Segundo Lysek e cols ${ }^{8}$, um fungo é caracterizado como ovicida se durante o processo de infecção dos ovos apresentar o efeito do tipo 3 (ação ovicida). Esse fato está de acordo com os resultados percentuais demonstrados pelos isolados fúngicos de Pochonia chlamydosporia no presente trabalho.

Em outros trabalhos, Braga cols ${ }^{13}$ mencionam que, tanto o isolado VC1 ou VC4 de Pochonia chlamydosporia poderiam ser utilizados no controle biológico de ovos de parasitos gastrintestinais, uma vez que têm demonstrado a mesma ação ovicida $(p>0,01)$ em condições laboratorias. No entanto, se faz necessário à realização de mais pesquisas que possam demonstrar a interação destes e de outros isolados fúngicos de Pochonia chlamydosporia sobre ovos de nematóides gastrintestinais de animais domésticos e de seres humanos. No presente trabalho, foram utilizados os isolados fúngicos $\mathrm{VC} 1$ e VC4 e outros isolados de Pochonia chlamydosporia apresentando a mesma ação ovicida $(\mathrm{p}>0,01)$ sobre os ovos de Toxocara canis.

O mecanismo de penetração dos fungos ovicidas nos ovos parasitados ainda não está totalmente elucidado, e por isso existem diferenças na sua atividade ovicida (Braga e cols ${ }^{14}$. Entretanto, vários autores admitem que a atividade enzimática seja um dos principais componentes no processo de ataque e penetração através dos ovos e por isso estes fungos poderiam ser utilizados no controle biológico ${ }^{15}$.

Dessa forma, por meio dos resultados percentuais demonstrados no presente trabalho, sugere-se a empregabilidade de Pochonia chlamydosporia como uma alternativa de controle biológico dos ovos embrionados de Toxocara canis eliminados nas fezes, e com isso interferindo diretamente no ciclo biológico deste parasito.

\section{CONFLITO DE INTERESSE}

Os autores declaram não haver nenhum tipo de conflito de interesse.

\section{SUPORTE FINANCEIRO}

Conselho Nacional de Desenvolvimento Científico e Tecnológico (CNPq), Coordenação de Aperfeiçoamento de Pessoal de Nível Superior (CAPES), Fundação de Amparo à Pesquisa do Estado de Minas Gerais (FAPEMIG).

\section{REFERÊNCIAS}

1. Vasconcellos MC, Barros JSL, Oliveira CS. Parasitas gastrointestinais em cães institucionalizados no Rio de Janeiro, RJ. Rev Saúde Públic 2006; 40: 321-323.

2. Barriga OO. A critical look at the importance, prevalence and control of toxocariasis and the possibilities of immunological control. Vet Parasitol 1998;29:295-234.

3. Urquhart GM, Armour J, Duncan JL, Dunn AM, Jennings FW. Parasitologia Veterinária. $2^{\text {rd }}$ edition Rio de Janeiro, Guanabara Koogan; 1988.

4. Taylor MRH, Keane CT, O’Connor P, Girdwood RWA, Smith H. Clinical features of covert toxocariasis. Scand J Infect Dis 1987;19:693-696.

5. Araujo JM, Araújo JV, Braga FR, Carvalho RO, Silva AR, Campos AK. Interaction and ovicidal activity of nematophagous fungus Pochonia chlamydosporia on Taenia saginata eggs. Experiment Parasitol 2009;121:338-341.
6. Braga FR, Araújo JV, Campos AK, Carvalho RO, Silva AR, Tavela AO, Maciel AS. Observação in vitro da ação dos isolados fúngicos Duddingtonia flagrans, Monacrosporium thaumasium e Verticillium chlamydosporium sobre ovos de Ascaris lumbricoides (Lineu, 1758). Rev Soc Bras Med Tropic 2007;40: 356-358.

7. Araújo JV, Santos MA, Ferraz S. Efeito ovicida de fungos nematófagos sobre ovos embrionados de Toxocara canis. Arq Bras Med Vet Zootec 1995;47:37-42.

8. Lysek H, Fassatiová O, Pineda NC, Hernández NL. Ovicidal fungi in soils of Cuba. Folia Parasitol 1982;29:265-270.

9. Ayres M, Ayres JM, Ayres DL, Santos, A.S. Aplicações estatísticas nas áreas de ciências biomédicas. Belém: Sociedade Civil Maniraua, 2003.

10. Santarém VA, Giuffrida R, Zanin GA. Larva migrans cutânea: ocorrência de casos humanos e identificação de larvas de Ancylostoma spp em parque público do município de Taciba, São Paulo. Rev Soc Bras Med Tropic 2004;37:179-181.

11. Bourdeau P. Toxocara canis: infestation du chien etde l'homme, méthodes de lutte. Point Vet 1986;18:551-564.

12. Overgaauw PAM. Aspects of Toxocara epidemiology: human toxocarosis. Critical Rev Microbiol 1997;23:215-231.

13. Braga FR, Araújo JV, Campos AK, Araujo JM, Silva AR, Carvalho RO, Correa $\mathrm{DN}$, Pereira CAJ. In vitro evaluation of the effect of the nematophagous fungi Duddingtonia flagrans, Monacrosporium sinense and Pochonia chlamydosporia on Schistosoma mansoni eggs. World J Microbiol Biotechnol 2008; 24: 2713-2716.

14. Braga FR, Araújo JV, Carvalho RO, Silva AR, Araujo JM, Tavela AO, Campos AK, Costa PR. Ovicidal effect of nematophagous fungi on Taenia taeniae formis eggs. World J Microbiol Biotechnol 2009;25:533-535.

15. Lysek H, Sterba J. Colonization of Ascaris lumbricoides eggs by the fungus Verticillium chlamydosporium Goddard. Folia Parasitol 1991; 38: 255-259. 